\title{
Trabalho de conclusão de curso de Odontologia: uma análise de perfil
}

\author{
Mayara Cavalcante Gomes Noronha*; Layana Bruna Vaz*; Francisca Tereza Coelho Matos**; Carlos \\ Alberto Monteiro Falcão**; Thiago Lima Monte**; Isabela Floriano** \\ * Graduada em Odontologia, Centro Universitário \\ Uninovafapi \\ ** Professor(a) Titular de Odontologia, Centro Universitário \\ Uninovafapi
}

Recebido: 12/07/2020. Aprovado: 18/01/2021.

\begin{abstract}
RESUMO
Este estudo avaliou o perfil de pesquisa acadêmica do Curso de Odontologia do Centro Universitário Uninovafapi por meio da análise de todos os Trabalhos de Conclusão de Curso (TCC) apresentados no período de 2014/1 a 2019/1. Trata-se de pesquisa documental de caráter exploratório realizada no repositório de TCC da instituição. Dos 267 TCC defendidos no período pesquisado, 262 atenderam aos critérios de inclusão. Os resultados apontaram que a nota média foi 9,3 $\pm 0,9$ pontos. Observou-se que a área mais escolhida pelos alunos foi a Ortodontia (17,2\%). Apenas $19(7,3 \%)$ foram publicados em periódicos científicos. Conclui-se que a Ortodontia é a especialidade que desperta maior interesse nos acadêmicos de Odontologia para escrita do seu trabalho de conclusão. Além disso, verificou-se a necessidade de reflexão do corpo docente e discente para a concretização de publicações cientificas ainda na graduação.
\end{abstract}

Descritores: Comunicação Acadêmica. Monografia. Pesquisa em Odontologia.

\section{INTRODUÇÃO}

O Curso de Bacharelado em Odontologia tem o Trabalho de Conclusão de Curso (TCC) como um componente curricular obrigatório, de acordo com as Diretrizes Curriculares Nacionais $(\mathrm{DCN})^{1}$. Sob orientação docente, ele deve ser direcionado a um tema da Odontologia.

Ainda de acordo com a estrutura curricular, a prática da pesquisa deve ser incentivada, o que motiva estudantes e professores a contribuírem com a produção cientifica nacional ${ }^{2}$. Além disso, o aprofundamento de um tema da Odontologia por meio da pesquisa e/ou do TCC é uma modalidade ativa de ensino-aprendizagem e, portanto, de formação do acadêmico, especialmente devido à visão de que trabalhos científicos aprimoram a conduta clínica e figuram como diferencial na inserção no mercado de trabalho $0^{3,4,5}$.

Aliado a isso, os estudantes desejam aumentar suas habilidades e destreza em determinada especialidade ainda na durante a graduação. Assim, alguns deles escolhem os 
docentes e/ou temáticas do seu TCC como forma de adquirir tal profundidade direcionada ${ }^{6,7}$.

Identificar as perspectivas e motivações dos acadêmicos de Odontologia diante do TCC é uma estratégia para saber quais medidas podem ser adotadas para o aperfeiçoamento dessa excelente metodologia de ensino-aprendizagem. Entretanto, não se sabe quais as áreas de maior interesse para elaboração do TCC, assim como quantos deles são efetivamente publicados na forma de artigos científicos. Diante disso, o objetivo do estudo foi fornecer informações básicas sobre o perfil de pesquisa acadêmica do Curso de Odontologia do Centro Universitário Uninovafapi.

\section{METODOLOGIA}

O Centro Universitário Uninovafapi é uma instituição de ensino superior (IES) localizada na cidade de Teresina, capital do Piauí, habilitada para ofertar o curso de Graduação de Bacharelado em Odontologia na modalidade presencial desde $2000^{8}$. A Resolução nº 05/2009 da IES normatiza o TCC e estabelece que sua elaboração seja iniciada no $6^{\circ}$ semestre do curso, deixando ao estudante a decisão de escolher o professor orientador de sua preferência ${ }^{9}$.

Em um primeiro momento, foram listados os TCCs defendidos por alunos regularmente matriculados no último período do curso de Odontologia de janeiro de 2014 a junho de 2019. Em seguida, eles foram acessados no repositório de TCCs da IES, assim como as notas atribuídas a eles após a defesa. Foram incluídos os TCCs cujo texto estava disponível na integra. Aqueles com texto indisponível, incompleto e/ou com nota ausente, zero ou reprovativa foram excluídos do estudo.

Cada TCC teve o texto lido por completo por dois examinadores, individualmente. Os trabalhos foram classificados quanto aos seguintes critérios: a) área de concentração (Anestesiologia e Terapêutica, Bioética e Odontologia Legal, Biossegurança e Microbiologia, Cirurgia e
Traumatologia, Dentística e Materiais Dentários, DTM e Dor Orofacial, Endodontia, Odontologia para Pacientes Especiais, Odontopediatria, Ortodontia, Patologia, Periodontia e Implantodontia, Prótese e Odontogeriatria, Radiologia, Saúde Pública, Outros); b) metodologia empregada (revisão sistemática, revisão de literatura, revisão integrativa, estudo clínico, estudo laboratorial, estudo transversal e relato de caso); e c) publicação ou não em periódicos científicos.

A verificação da publicação dos TCCs em periódicos científicos foi feita por meio da pesquisa dos títulos nas bases de dados SciELO, PubMed e Google Acadêmico e/ou diretamente no Currículo Lattes dos docentes e discentes envolvidos.

Os dados coletados foram tabulados $\mathrm{e}$ analisados descritivamente no software Microsoft Excel ${ }^{\circledR}$ (Microsoft Corporation, Redmond, EUA). Tabelas e gráficos foram elaborados no mesmo software.

\section{RESULTADOS}

Dos 267 TCCs defendidos, foram incluídos 262, pois quatro não tiveram o texto completo encontrado e um recebeu nota zero. A nota dos trabalhos foi, em média, 9,3 $\pm 0,9$ pontos.

A tabela 1 mostra a classificação dos TCCs conforme a área da Odontologia. Observou-se que a área mais escolhida pelos alunos foi a Ortodontia, seguida de Periodontia, Implantodontia, Dentística e Materiais Dentários. As áreas de Anestesiologia e Terapêutica, DTM e Dor Orofacial e Radiologia apresentaram as menores quantidades de trabalhos.

A análise metodológica revelou que a maior parte da produção analisada era de revisões de literatura (figura 1). Além disso, observou-se que apenas 7,3\% dos TCCs foram publicados em periódicos científicos. Entre os que foram publicados, predominaram revistas de estratos Qualis Capes baixos, principalmente B3 e B4 (figura 2). 
Tabela 1. Distribuição dos TCCs incluídos conforme área do conhecimento da Odontologia

\begin{tabular}{ll}
\hline \multicolumn{1}{c}{ ÁREA } & n (\%) \\
\hline Ortodontia & $45(17,2)$ \\
Periodontia e Implantodontia & $41(15,7)$ \\
Dentística e Materiais Dentários & $39(14,9)$ \\
Odontopediatria & $17(6,5)$ \\
Endodontia & $16(6,1)$ \\
Cirurgia & $16(6,1)$ \\
Patologia & $15(5,7)$ \\
Pacientes Especiais & $13(5,0)$ \\
Biossegurança e Microbiologia & $12(4,6)$ \\
Saúde Pública & $11(4,2)$ \\
Prótese e Odontogeriatria & $9(3,6)$ \\
Outros & $9(3,6)$ \\
Bioética e Odontologia Legal & $8(3,1)$ \\
Radiologia & $4(1,5)$ \\
Anestesiologia e Terapêutica & $4(1,5)$ \\
DTM e Dor orofacial & $3(1,1)$ \\
\hline
\end{tabular}
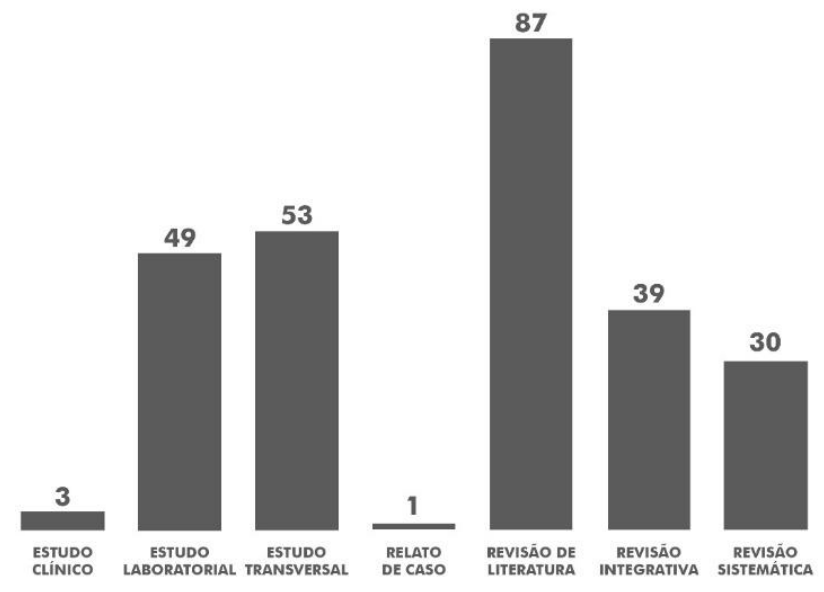

Figura 1. Distribuição dos TCCs incluídos quanto ao tipo de estudo

\section{DISCUSSÃO}

No presente estudo a Ortodontia foi a área que apresentou maior número de trabalhos. A literatura aponta que acadêmicos de outros países têm preferência semelhante ${ }^{10,6}$. Isso pode ser devido a dois fatores. Um deles, a pequena carga horária de Ortodontia nos currículos de graduação faz com que sejam abordados apenas conhecimentos iniciais da área. Diante disso, a

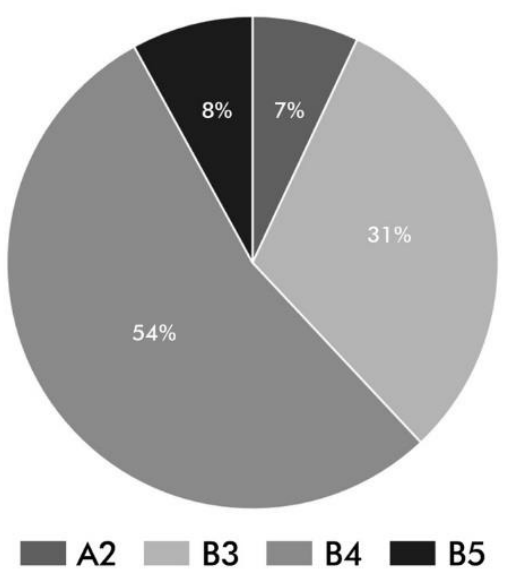

Figura 2. Classificação Qualis Capes das revistas nas quais os TCCs foram publicados

especialidade passa a despertar a curiosidade dos alunos, que a escolhem como tema de TCC como uma forma de ter mais contato com ela. $\mathrm{O}$ outro fator, a associação da especialidade com cirurgiões-dentistas bem-sucedidos profissional e financeiramente, assim como casos clínicos com resoluções clínicas de impacto visual.

A preferência dos estudantes por determinadas áreas do conhecimento reflete uma 
pretensão de pós-graduação, afinidade e/ou facilidade de trabalhar com docentes dessas especialidades. Prova disso é que no Brasil, a Ortodontia é a especialidade com mais cirurgiõesdentistas registrados no Conselho Federal de Odontologia $^{12}$. Cabe ressaltar que essa área está associada à maior renda e, portanto, fornece status e benefícios financeiros que aumentam sua atratividade para discentes ${ }^{13}$

Especificamente no Centro Universitário Uninovafapi, apenas duas especialidades são ofertadas como cursos de pós-graduação lato sensu e as referidas áreas são também as mais escolhidas pelos discentes como área do TCC: Ortodontia e Implantodontia. Característica semelhante foi encontrada com os estudantes da Universidade Federal de Pelotas ${ }^{11}$.

Com relação à Periodontia e à Dentística, entende-se que se destacam na escolha dos alunos por serem áreas do conhecimento que o acadêmico de Odontologia trabalha praticamente durante o curso inteiro. Isto é, são unidades curriculares que logo no segundo ano de curso são ministradas de forma teórico-laboratorial e seguem sendo trabalhadas de forma clínica até o final da graduação. Realidade similar é encontrada em outros países ${ }^{4}$.

O principal método de pesquisa utilizado como TCC foi a revisão de literatura, que reúne a literatura científica publicada sobre determinado tema de forma não sistematizada e até, de certa forma, parcial. Alguns acadêmicos afirmam que o tempo que dedicam à elaboração do TCC é curto e isso favorece a adoção dessa metodologia, que é mais simples. Realmente, estudos de revisão sistemática e estudos clínicos randomizados, sabidamente mais relevantes para a ciência, são pouco realizados por alunos de graduação ${ }^{14}$. A divulgação de manuscritos de discentes de graduação para a comunidade científica é uma forma importante de iniciar uma carreira de pesquisa. Além disso, a dinâmica de escrita e submissão de artigos em periódicos é uma forma de inserir ativamente o discente na ciência. Tal fato independe do nível de qualidade e relevância dos periódicos escolhidos. Relatório recente mostrou que no Brasil houve um crescimento significativo no número de trabalhos publicados entre 20132018 e os cursos de graduação de instituições de ensino superior foram peça fundamental para esse resultado ${ }^{15}$.

Acredita-se que a pequena taxa de publicação dos TCCs analisados em periódicos científicos ocorra porque o formato do TCC defendido é o formato institucional, isto é, para ser arquivado no repositório da IES. Não necessariamente significa que são trabalhos de qualidade inferior, visto que as notas atribuídas pela banca examinadora foram altas.

Como a defesa ocorre no último período do curso, a maioria dos estudantes não dão sequência à adaptação da escrita do trabalho no formato de artigo científico e, por consequência, não o submetem para publicação. Por conta disso, a maioria dos trabalhos desenvolvidos na IES ficam guardados no acervo da instituição e não são enviados como publicação científica para divulgação aos pares. É interessante, portanto, repensar o formato de defesa do TCC da IES para que, ainda durante o curso, o mesmo seja submetido como artigo científico para publicação.

Dentre as características positivas que podem ser notadas nos acadêmicos que participam de projetos científicos é maior autonomia, resolutividade de problemas e melhor desempenho na carreira profissional ${ }^{16}$. Entretanto, o presente estudo não analisou as facilidades e dificuldades na elaboração dos Trabalhos de Conclusão de Curso na graduação de Odontologia, área importante a ser pesquisada.

\section{CONCLUSÃO}

A Ortodontia foi a especialidade que despertou maior interesse nos acadêmicos de 
Odontologia pesquisados para escrita do TCC. Além disso, poucos TCCs são efetivamente publicados em periódicos científicos.

\section{AGRADECIMENTOS}

Agradecemos à equipe que compõe a Biblioteca do Centro Universitário Uninovafapi pelo apoio na condução do presente estudo.

\section{ABSTRACT}

Dentistry course monograph: a profile analysis This study analyzed the academic research profile of the Dentistry Course at Uninovafapi University Centre through the analysis of all monographs presented in the period from 2014.1 to 2019.1. This is an exploratory research carried out in institution's monograph repository. The collected data were tabulated and analyzed with Microsoft Excel ${ }^{\circledR}$ software. Of the 267 monographs, 262 were included. The results showed that mean grade was $9.3 \pm 0.9$ points. It was observed that the area most chosen by students is Orthodontics (17.2\%). Only 19 were published in scientific journals. We conclude that Orthodontics is the specialty with the greatest interest. In addition, it is important for teaching staff and students to think about achieving scientific publications during the undergraduate course.

Descriptors: Academic Communication. Monography. Research in Dentistry.

\section{REFERÊNCIAS}

1. Conselho Nacional de Educação (Brasil). Resolução $n^{\circ}$. 3, de 19 de fevereiro de 2002. Institui Diretrizes Curriculares Nacionais do Curso de Odontologia. Diário Oficial da União 4 mar 2002; Seção 1, p.10.

2. Cavalcanti AL, Melo TSNB, Barroso KMA, Souza FEC, Maia AMA, Silva ALO. Perfil da pesquisa científica em Odontologia realizada no Brasil. Pesq Bras Odontoped Clin Integr. 2004;4(2):99-104.

3. Cavalcanti AL. Introdução à pesquisa aplicada à Odontologia: bases para a iniciação científica. Publicatio UEPG. 2003;9(3/4):45-53.

4. Silva-Junior MF, Assis RIF, Sousa HA, Miclos PV, Gomes MJ. Iniciação científica: percepção do interesse de acadêmicos de Odontologia de uma universidade brasileira. Saúde Soc. 2014;23(1):325-35.

5. Tachibana M, Pavani R, Bariani IC. Participação em eventos científicos e formação do universitário. Psico. 2004;35(1):89-96.

6. Rashid HH, Ghotane SG, Abufanas SH, Gallagher JE. Short and long-term career plans of final year dental students in the United Arab Emirates. BMC Oral Health. 2013;13:39-48.

7. Gallagher JE, Patel R, Wilson NHF. The emerging dental workforce: long-term career expectations and influences. A quantitative study of final year dental students' views on their long-term career from one London Dental School. BMC Oral Health. 2009;9:35-44.

8. Brasil. Ministério da Educação. Portaria Ministerial $n^{\circ} 1.592$ de 09 de outubro de 2000.Diário Oficial da União, Brasília, 13 de outubro de 2000.

9. Uninovafapi. Projeto pedagógico do curso de bacharelado em odontologia. Teresina, PI: Uninovafapi, 2015.

10. James P, Veselina K, Alisa K. Final-year dental undergraduate attitudes towards specialisation. Dent J. 2016;4(3):26-36.

11. Chisini LA, Grehs HS, Nóbrega KHS, Conde MCM, Corrêa MB, Dermarco FF. Análise descritiva dos trabalhos de conclusão de curso da faculdade de Odontologia UFPel. Rev ABENO. 2017;17:8-15.

12. Conselho Federal de Odontologia. Quantidade Geral de Cirurgiões-Dentistas Especialistas. 2021 [Acesso em: 
18/03/2021]. Disponível em: funding excellence. Web of Science Group https://website.cfo.org.br/estatisticas/quanti [Internet]. [Acesso em: 01/05/2016]. dade-geral-de-cirurgioes-dentistas- Disponível em: https://jornal.usp.br/wpespecialistas/.

13. Cordes DW, Doherty N, Lopez R. Assessing the economic return of specializing in orthodontics or oral and maxillofacial surgery. J Am Dent Assoc. 2001;132(12):1679-84.

14. OCEBM Levels of Evidence Working Group. The Oxford 2011 Levels of Evidence. Oxford Centre for EvidenceBased Medicine [Internet]. 2016 May [Acesso em: 01/05/2016]. Disponível em: http://www.cebm.net/index.aspx?o=5653.

15. Web of Science Group. Research in Brazil: content/uploads/2019/09/ClarivateReport_2 013-2018.pdf.

16. Fava MF, Fava M. A iniciação científica: muitas vantagens e poucos riscos. São Paulo Perspec. 2000;14(1):73-7.

\section{Correspondência para:}

Isabela Floriano

e-mail: isabela.floriano@uninovafapi.edu.br Curso de Odontologia

Rua Prof. Vitorino Orthiges Fernandes, 6123 Uruguai

64073-505 Teresina/PI 